04

\title{
Метод формирования единственного сфокусированного порядка дифракции при помощи бинарных амплитудных дифракционных элементов без пространственной несущей
}

\author{
(C) В.В. Краснов, Р.С. Стариков, Е.Ю. Злоказов \\ Национальный исследовательский ядерный университет „МИФИ“, \\ 115409 Москва, Россия \\ e-mail: vitaly.krasnov@mail.ru
}

Поступила в редакцию 16.12.2020 г.

В окончательной редакции 16.12.2020 г.

Принята к публикации 22.12.2020 г.

Предложен метод синтеза самофокусирующихся амплитудных дифракционных оптических элементов (ДОЭ) без несущей пространственной частоты для работы в расходящихся пучках и формирующих единственный сфокусированный порядок дифракции, который может занимать всю область восстанавливаемого изображения ДОЭ ввиду отсутствия необходимости пространственного разделения порядков. Синтез проходит в два этапа. Первый осуществляется итерационным алгоритмом, подобным алгоритму Герчберга-Сэкстона, с теми отличиями, что синтезируемый ДОЭ амплитудный, а не фазовый, падающий волновой фронт - расходящийся сферический. Далее применяется метод прямого поиска со случайной траекторией. В результате для бинарного амплитудного ДОЭ удалось достичь значений ошибки синтеза в 6\% и дифракционной эффективности 6\%. Представлены результаты экспериментальной реализации ДОЭ с использованием микрозеркального модулятора света.

Ключевые слова: амплитудный ДОЭ, единственный порядок дифракции, расходящийся пучок, синтез ДОЭ, итерационный алгоритм, микрозеркальный модулятор света.

DOI: $10.21883 /$ OS.2021.04.50771.292-20

\section{Введение}

Дифракционные оптические элементы (ДОЭ) широко используются в различных областях науки и техники [1-4]. Популярной разновидностью ДОЭ являются голограммы, которые могут быть получены как оптическими методами, так и численными (компьютерносинтезированные голограммы), могут быть реализованы в виде как амплитудных, так и фазовых ДОЭ [5]. Однако голограммам свойственны определенные недостатки. Как правило, голограммы являются результатом интерференции опорного и объектного пучков, в результате чего имеется несущая пространственная частота, приводящая к формированию нескольких порядков дифракции, соответственно необходимо обеспечить пространственное разделение этих порядков. Кроме того, голограммы отличаются достаточно низкой дифракционной эффективностью, так, например, бинаризованные амплитудные голограммы имеют дифракционную эффективность порядка 2-4\% [6].

Существует разновидность ДОЭ, которые свободны от указанных недостатков, - киноформы $[1,7,8]$. Это фазовые ДОЭ без несущей пространственной частоты, которые формируют единственный порядок дифракции при освещении их плоской волной. Аналитического решения задачи синтеза киноформа в общем виде не существует, синтез осуществляется итерационными методами, обеспечивающими сравнительно небольшую ошибку синте- за [9]. Поскольку киноформы - полностью фазовые элементы, их невозможно использовать со сверхбыстрыми микрозеркальными (МЗ) пространственно-временными модуляторами света (ПВМС) [10-13] ввиду бинарного амплитудного характера модуляции.

Целью данной работы является разработка метода и создание на его основе амплитудных дифракционных

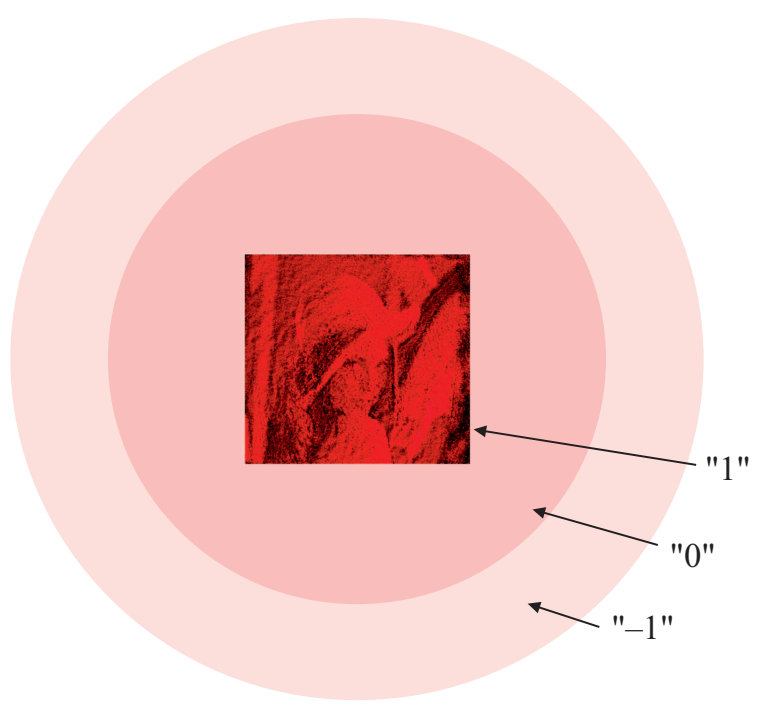

Рис. 1. Схематичное распределение порядков дифракции новых амплитудных ДОЭ без несущей пространственной частоты. 


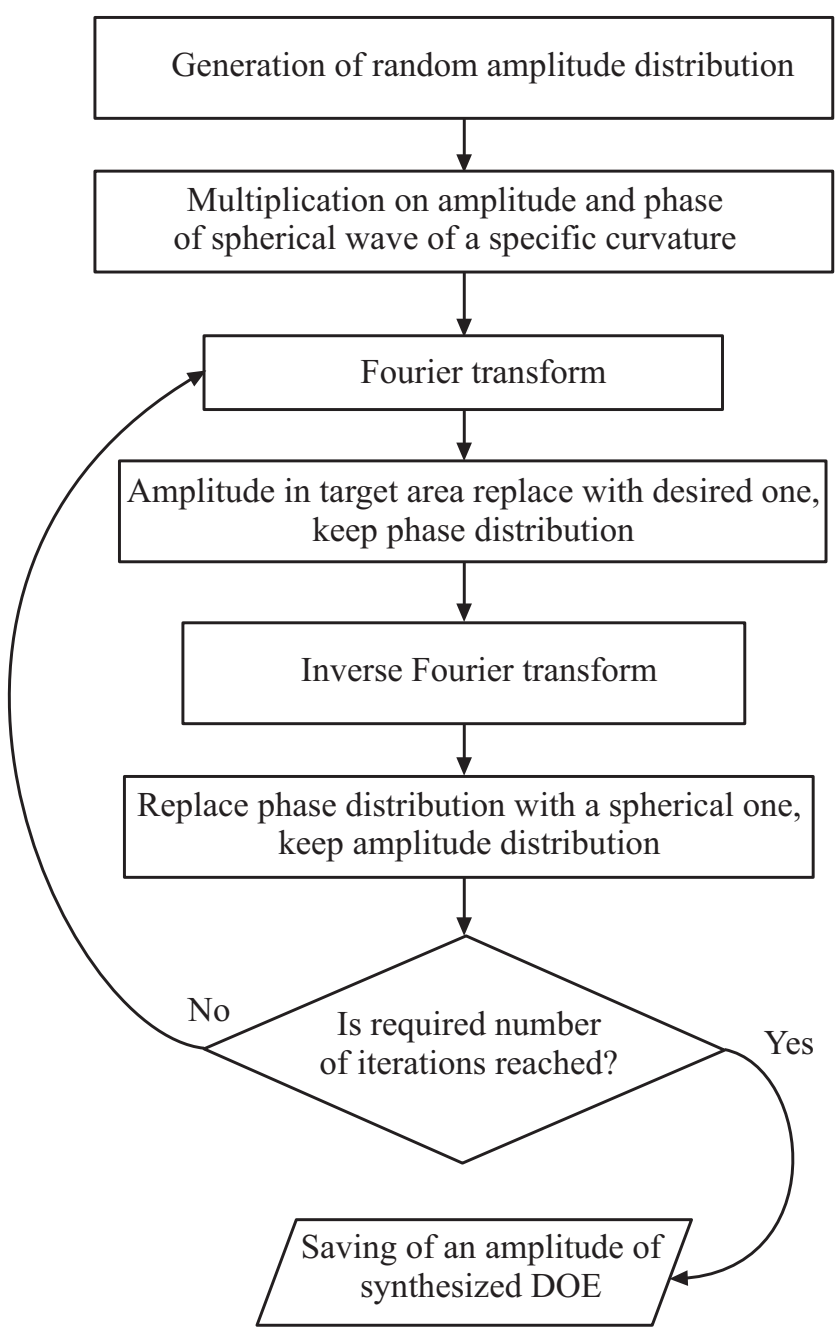

Рис. 2. Блок-схема алгоритма первого этапа синтеза амплитудных ДОЭ нового типа.

элементов нового типа - не имеющих несущей пространственной частоты и формирующих единственный сфокусированный порядок дифракции подобно киноформам, подходящих при этом для вывода на микрозеркальные модуляторы света.

\section{1. Метод синтеза новых самофокусирующихся амплитудных ДОЭ без несущей пространственной частоты}

Для синтеза новых амплитудных ДОЭ без несущей пространственной частоты был разработан итерационный алгоритм. Для того чтобы получить единственный сфокусированный порядок дифракции, данные ДОЭ должны освещаться расходящейся волной определённого радиуса кривизны. Таким образом, требуемое световое распределение сформируется в первом порядке дифракции на определенном расстоянии от ДОЭ (расчет в приближении Френеля) или на бесконечности (расчет в приближении Фурье). При этом электромагнитное поле в нулевом и минус первом порядках дифракции оказывается распределенным по большой области, создавая незначительную фоновую засветку. Схематичное расположение порядков дифракции иллюстрируется на рис. 1. Следует отметить сходства предлагаемого подхода со схемой осевой голографии в зоне дифракции Френеля, которые заключаются в соосном распространении порядков дифракции, отсутствии фокусирующей оптики и формировании сфокусированного первого порядка дифракции, в то время как минус первый оказывается расфокусированным. Отличия же заключаются, во-первых, в освещении ДОЭ расходящейся, а не плоской волной, что приводит к тому, что нулевой порядок также является расходящейся волной, рассеиваясь по большой площади, в то время как минус первый претерпевает еще большее

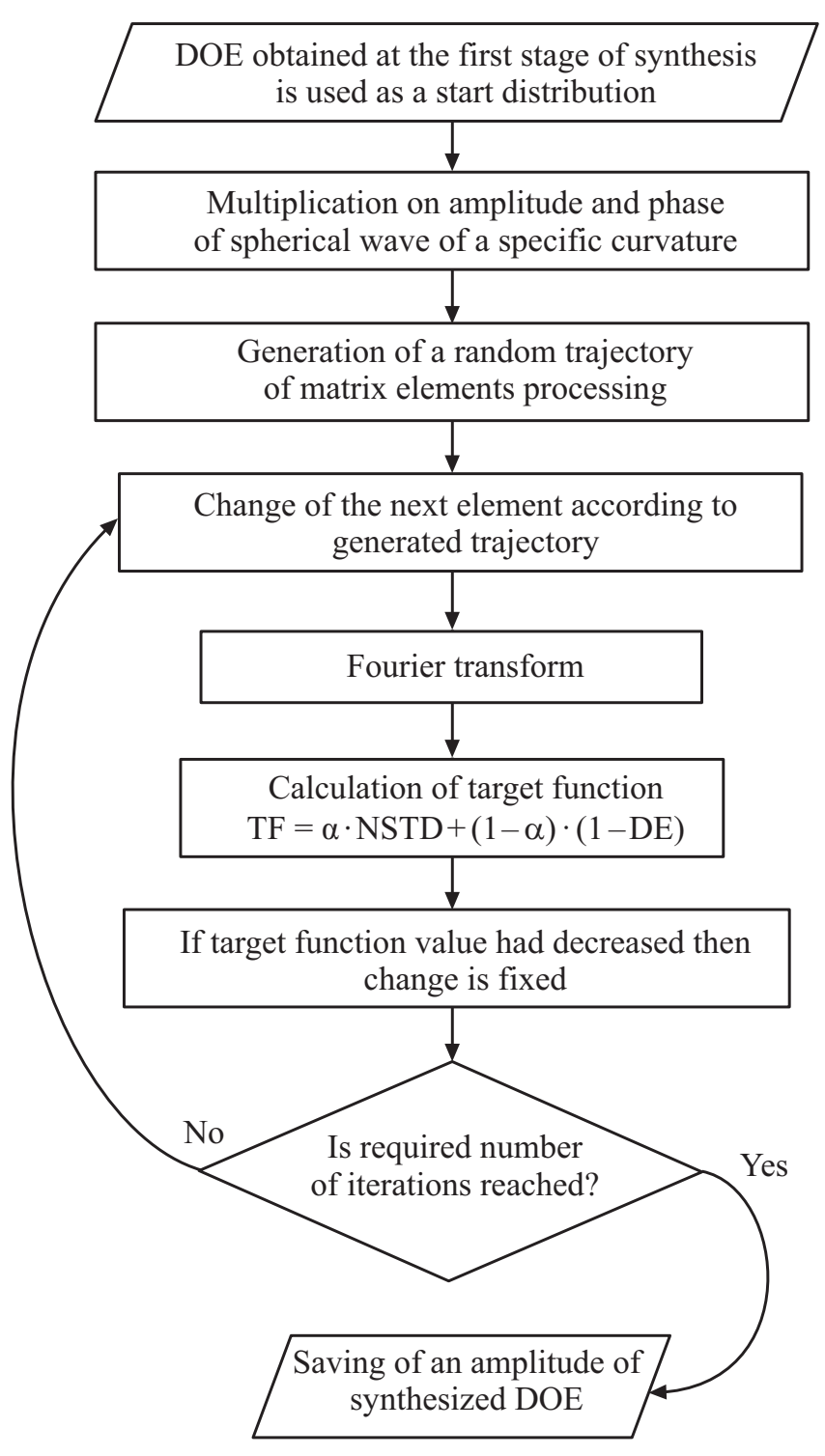

Рис. 3. Блок-схема алгоритма второго этапа синтеза амплитудных ДОЭ нового типа. 

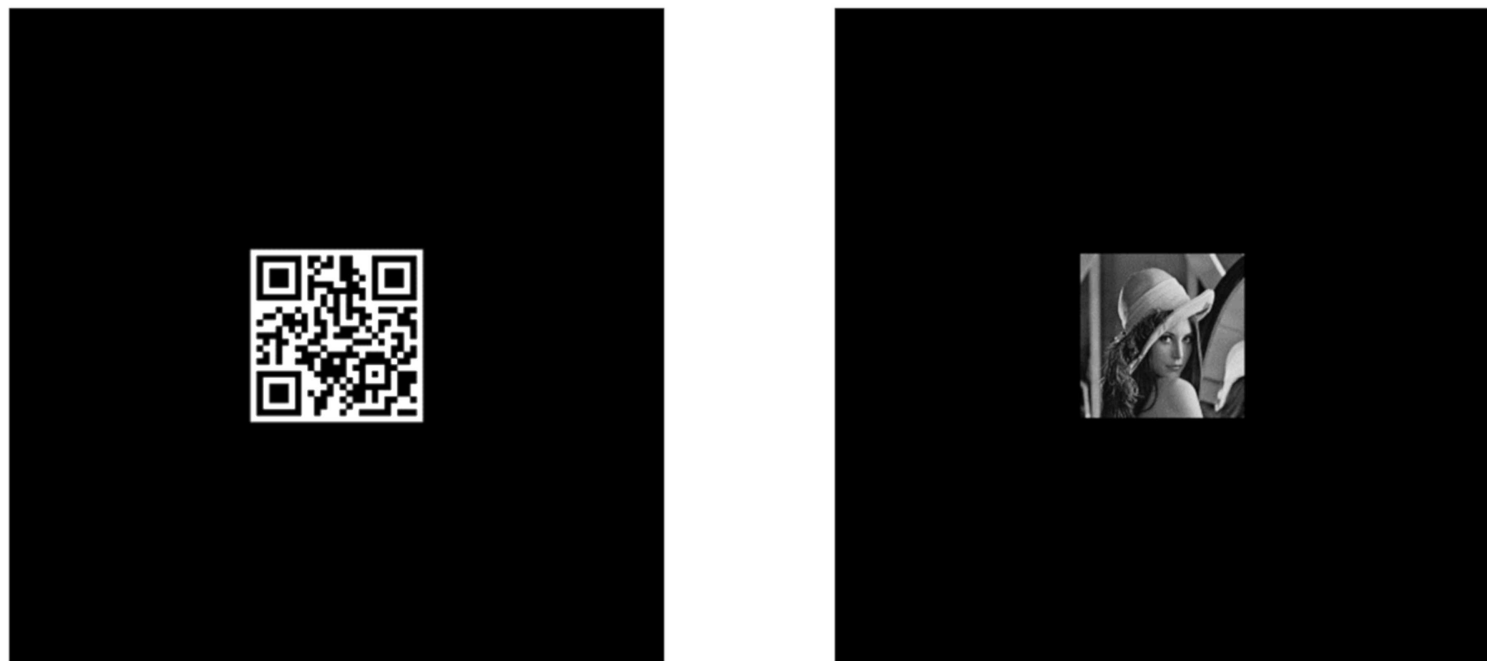

Pис. 4. Расчетная область восстанавливаемого изображения с тестовыми изображениями: бинарное QR-код (слева) и полутоновое „Лена“ (справа).

рассеяние. И, во-вторых, в оригинальном итерационном методе синтеза, схожем с методами синтеза безопорных фазовых ДОЭ - киноформов [1,7,8].

Синтез ДОЭ нового типа осуществлялся в два этапа. Первый - предварительный, идеологически основан на методе Герчберга-Сэкстона [14]. Блок-схема алгоритма приведена на рис. 2. Сперва, в качестве первого приближения ДОЭ, генерируется случайная амплитуда, которая умножается на амплитуду и фазу сферической волны заданного радиуса. Затем посредством преобразования Френеля переходим в плоскость изображения. Здесь амплитуду получившегося светового распределения заменяем на требуемую, а фазу сохраняем. Выполняем обратное преобразование. В получившемся амплитуднофазовом распределении фазу заменяем сферической, а амплитуду сохраняем. Далее повторяем эти шаги до тех пор, пока не будет выполнено заданное число итераций. Необходимое число итераций определяется по наступлению стагнации значения целевой функции, в роли которой выступает суперпозиция нормированного среднеквадратического отклонения и дифракционных потерь. В конце осуществляем бинаризацию получившегося ДОЭ. На этом завершается первый этап синтеза и начинается второй.

Второй этап нацелен на снижение ошибки синтеза по результатам первого этапа и включает применение метода прямого поиска со случайной траекторией $[15,16]$. Блок-схема алгоритма приведена на рис. 3. Элементы ДОЭ поочередно изменяются в соответствии со случайной траекторией обхода (в случае бинарных ДОЭ, если значение элемента было равно нулю, ему присваивается единица, если единице - ноль), при снижении значения целевой функции изменение сохраняется, в противном случае - отбрасывается.

В качестве целевой функции (ЦФ) была использована суперпозиция нормированного среднеквадратического отклонения (НСКО) [17] восстановленного изображения от исходного и дифракционной эффективности (ДЭ):

$$
\text { ЦФ }=\alpha \mathrm{HCКО}+(1-\alpha)(1-\text { ДЭ }),
$$

где $\alpha-$ весовой коэффициент. НСКО определялось как

$$
\text { НСКО }=\sqrt{1-\frac{\left(\sum_{\xi, \eta=1}^{M, N} E[\xi, \eta] F[\xi, \eta]\right)^{2}}{\left(\sum_{\xi, \eta=1}^{M, N} E^{2}[\xi, \eta]\right)\left(\sum_{\xi, \eta=1}^{M, N} F^{2}[\xi, \eta]\right)}},
$$

где $E$ и $F-$ оригинальное и восстановленное изображения соответственно. Дифракционная эффективность определялась как доля световой энергии, пришедшая в область формирования информативного первого порядка дифракции, по отношению к полной энергии, падающей на ДОЭ. Следует отметить, что данная оценка дает не точное значение дифракционной эффективности, а приближенное, так как в область первого порядка дифракции попадают также неинформативный нулевой и минус первый. Однако в случае, когда площадь, по которой рассеяны неинформативные порядки дифракции, много больше площади информативного порядка, этот эффект дает несущественную прибавку к величине дифракционной эффективности.

\section{2. Результаты экспериментов по синтезу ДОЭ нового типа}

В качестве тестовых изображений использовались: бинарное изображение QR-кода размером $270 \times 270$ отсчетов и полутоновое изображение „Лена“ размером $256 \times 256$ отсчетов. Изображения помещались по центру области восстановления размером $1024 \times 1024$ отсчетов (рис. 4). Синтез ДОЭ производился с использованием 2 градаций яркости с целью их последующего 

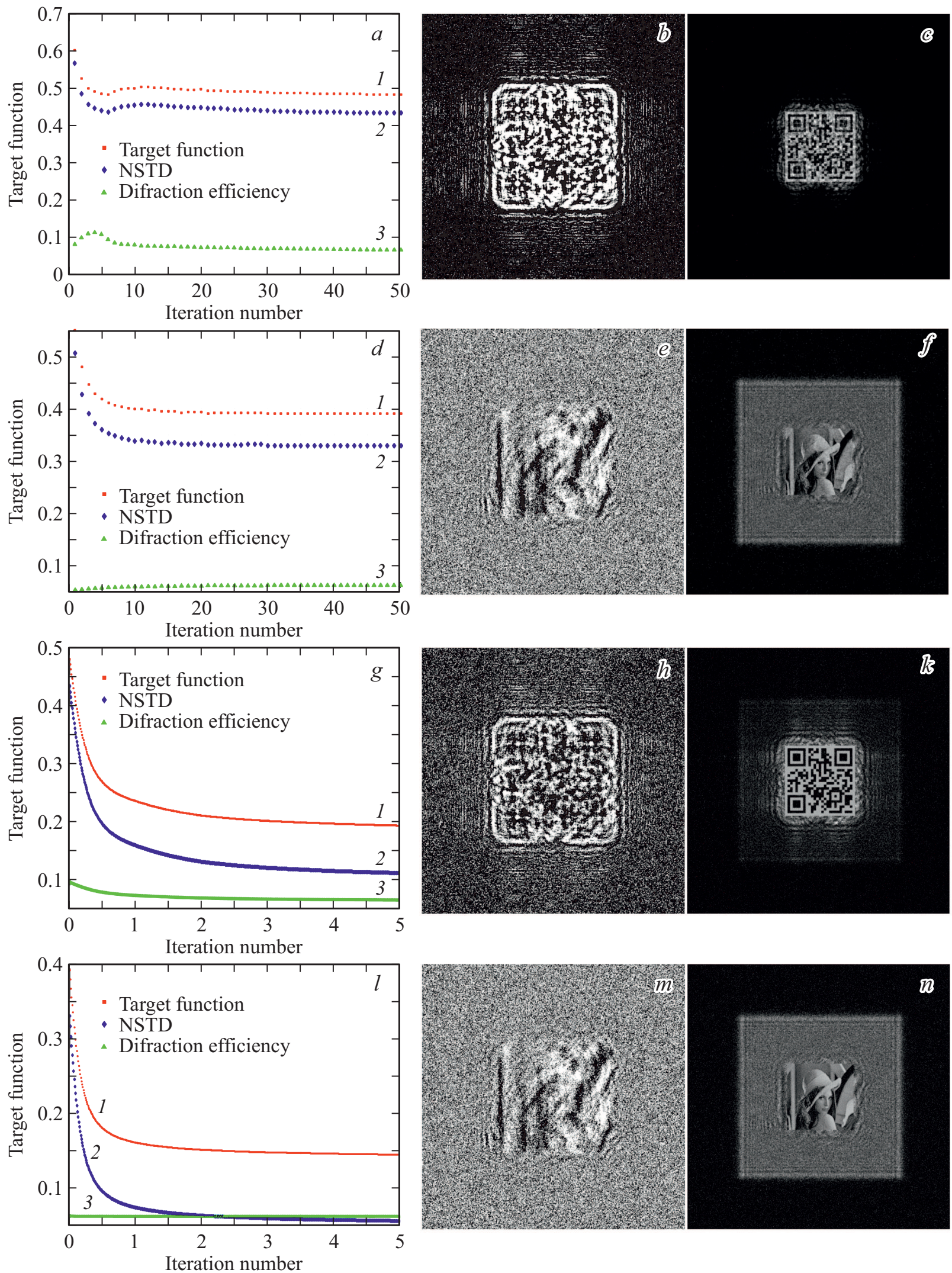

Рис. 5. Результаты первой $(a-f)$ и второй $(g-n)$ фаз синтеза бинарного амплитудного ДОЭ нового типа: зависимости целевой функции $(1)$, НСКО (2) и дифракционной эффективности (3) от номера итерации $(a, d, g, l)$, ДОЭ $(b, e, h, m)$ и восстановленные с ДОЭ изображения $(c, f, k, n)$. 

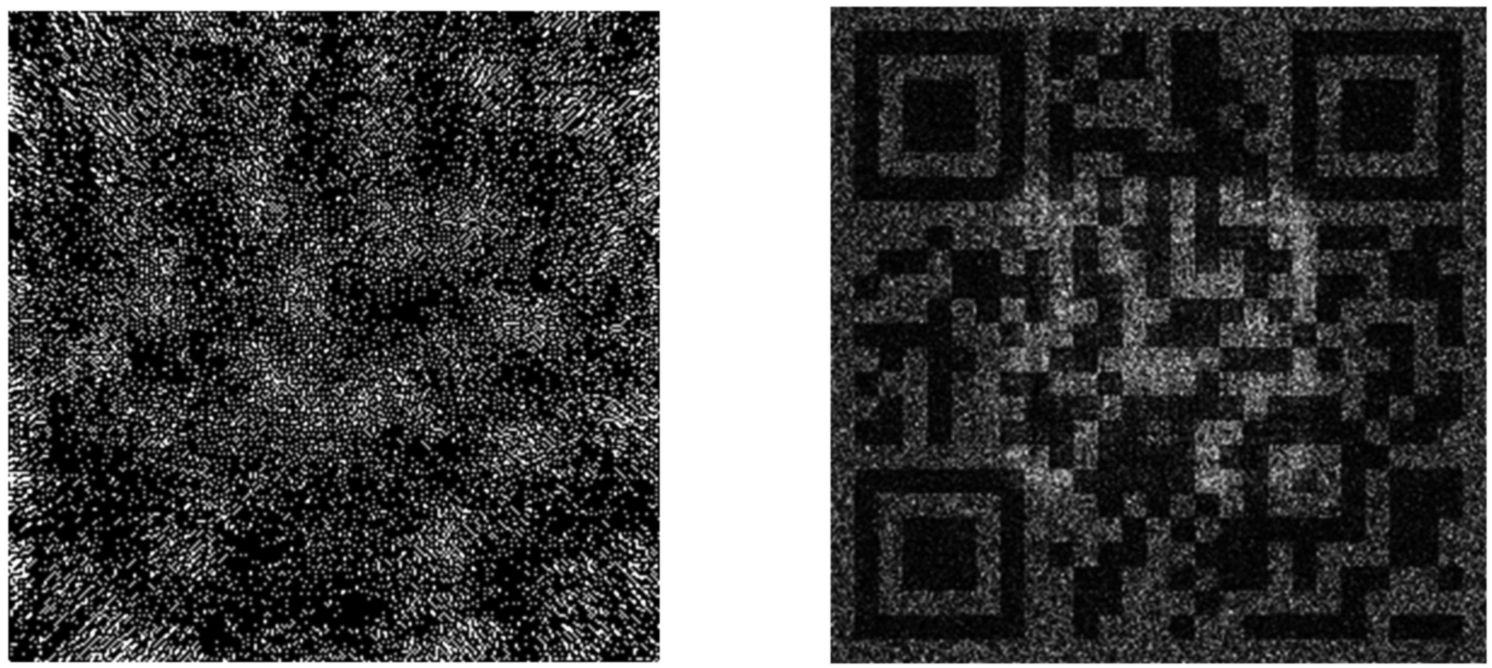

Рис. 6. Синтезированный бинарный амплитудный ДОЭ размером $270 \times 270$ отсчетов (слева) и восстановленное с него изображение (справа).

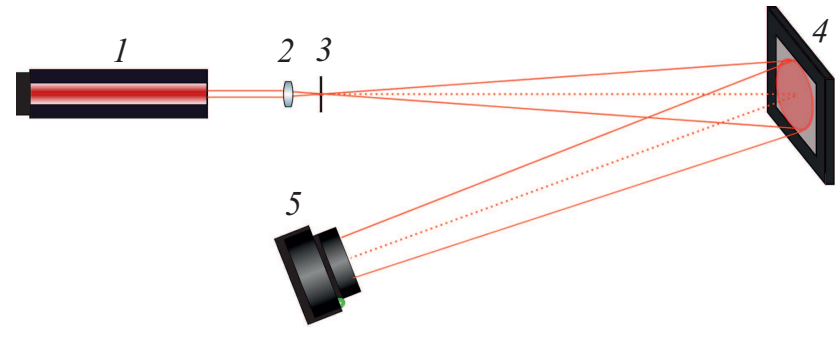

Рис. 7. Схема экспериментальной установки для оптического восстановления изображений с амплитудных бинарных ДОЭ без пространственной несущей: 1 - лазер, 2 - микрообъектив, 3 - микроапертура, 4 - МЗ ПВМС, 5 - камера.

вывода на МЗ ПВМС. Несмотря на то, что сами ДОЭ - бинарные, они способны формировать полутоновые изображения. Фокусное расстояние, равное радиусу кривизны волнового фронта, падающего на ПВМС, равнялось $30 \mathrm{~cm}$.

Весовой коэффициент $\alpha$ был выбран 0.9 с целью минимизации шумов синтеза. Зависимости НСКО, дифракционной эффективности и целевой функции от числа итераций первой фазы синтеза для обоих изображений представлены на рис. 5,a,d. Результаты первой фазы синтеза бинарного амплитудного ДОЭ нового типа представлены на рис. 5, $b, e$. Минимальные значения НСКО составили 0.44 и 0.33 для бинарного и полутонового изображений соответственно. При этом дифракционная эффективность составляла 9.5 и $6.3 \%$ для бинарного и полутонового изображений соответственно.

Результаты численного восстановления изображений с ДОЭ представлены на рис. 5, c,f. В случае полутонового изображения можно отчетливо наблюдать квадрат расфокусированного нулевого порядка.
Вторая фаза синтеза осуществлялась с использованием метода прямого поиска со случайной траекторией (ППСТ) [15,16]. В качестве стартового распределения использовались ДОЭ, полученные в результате первой фазы синтеза. Зависимости НСКО, дифракционной эффективности и целевой функции от числа итераций для второй фазы синтеза представлены на рис. $5, g, l$. В результате второй фазы синтеза НСКО снизилось с 0.44 до 0.11 для бинарного изображения и с 0.33 до 0.06 для полутонового. При этом дифракционная эффективность снизилась с 9.5 до 6.4\% для бинарного изображения и не изменилась для полутонового. Бинарные амплитудные ДОЭ нового типа, полученные в результате второй фазы синтеза, представлены на рис. 5, h, m. Результаты численного восстановления изображений с ДОЭ приведены на рис. $5, k$, $n$. В результате применения метода ППСТ получено снижение НСКО в 4-6 раз при падении дифракционной эффективности до 33\% по сравнению с результатами первой фазы синтеза.

Для демонстрации возможности использования всей области восстанавливаемого изображения ДОЭ был осуществлен синтез ДОЭ с фокусным расстоянием $10 \mathrm{~cm}$, содержащего изображение QR-кода размером $270 \times 270$ отсчетов на поле такого же размера. Результат синтеза и восстановленное изображение приведены на рис. 6. НСКО составило 0.56, дифракционная эффективность - 20\%. Высокое значение НСКО обусловлено использованием всей области восстанавливаемого изображения и соответственно отсутствием неинформативной области (т.е. области поля восстанавливаемого изображения ДОЭ, не занятого изображением), куда обычно осуществляется „сброс“ шума.

Таким образом, были синтезированы амплитудные бинарные ДОЭ, обладающие малой ошибкой синтеза на уровне 0.1 НСКО, а также высокой дифракционной 

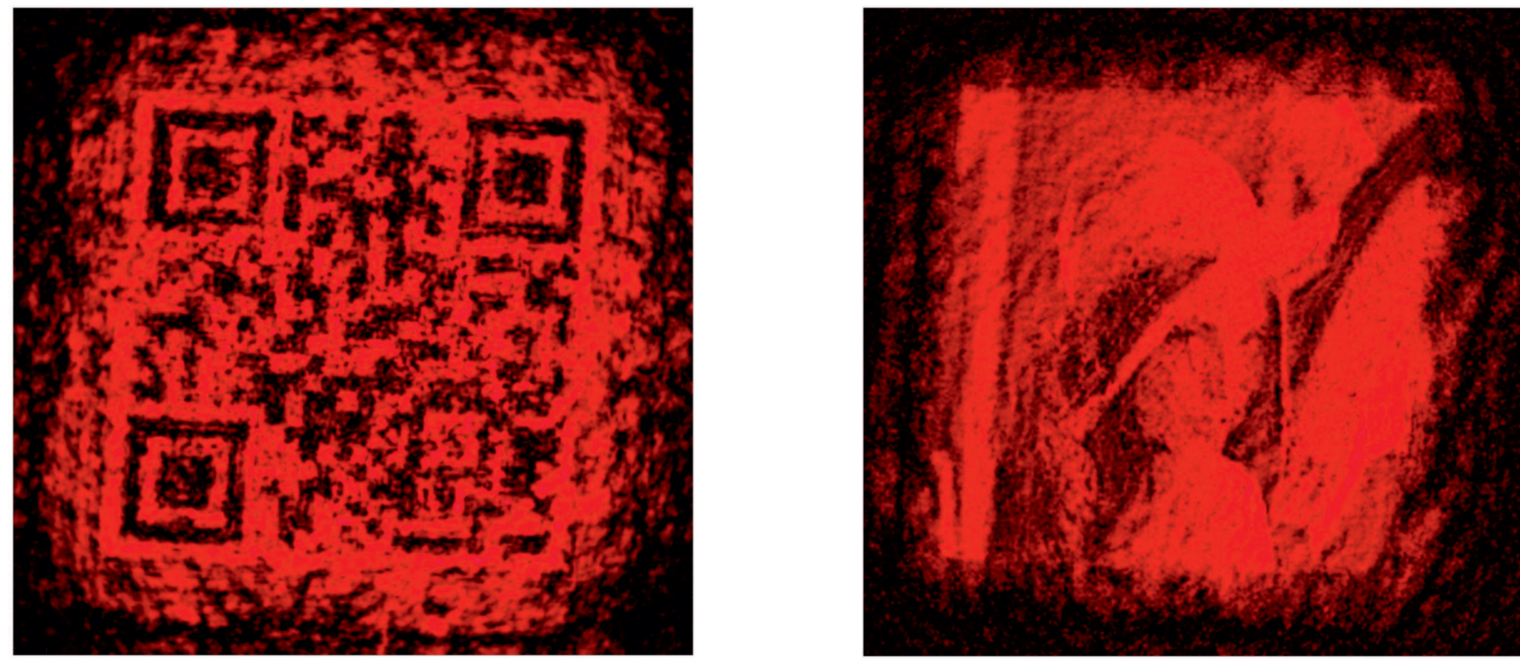

Рис. 8. Результат оптического восстановления изображений с бинарных амплитудных ДОЭ нового типа.

эффективностью, в среднем в 2 раза превышающей таковую у бинаризованных голограмм - на уровне $6.5 \%$, формирующих единственный сфокусированный порядок дифракции, который может занимать всю область восстанавливаемого изображения.

\section{3. Результаты экспериментов по оптическому восстановлению изображений с ДОЭ нового типа с использованием микрозеркального модулятора света}

Схема экспериментальной установки для оптического восстановления изображений с амплитудных бинарных ДОЭ без пространственной несущей приведена на рис. 7.

Источник излучения - гелий-неоновый лазер с длиной волны $633 \mathrm{~nm}$ и мощностью излучения $10 \mathrm{~mW}$. Излучение лазера фокусируется микрообъективом в микроапертуру. На расстоянии $40 \mathrm{~cm}$ от микроапертуры расположен М3 ПВМС Texas Instruments Discovery DLP9500BFLN (разрешение $1920 \times 1080$ пикселов, размер пиксела $10.8 \times 10.8 \mu \mathrm{m}$, частота смены кадров до $23 \mathrm{kHz}$ ), на котором отображается синтезированный ДОЭ. Камера Canon EOS M100, расположенная на расстоянии $80 \mathrm{~cm}$ от МЗ ПВМС, регистрирует оптически восстановленное с ДОЭ изображение.

Было произведено оптическое восстановление изображений с бинарных амплитудных ДОЭ нового типа размером $1024 \times 1024$ отсчетов, представленных на рис. $5, h, m$. Результаты оптического восстановления представлены на рис. 8. Несмотря на достаточно сильный спекл-шум (никаких мер для его подавления не применялось), восстановленные изображения хорошо идентифицируются с исходными (рис. 4).

\section{Заключение}

В работе предложен метод синтеза амплитудных дифракционных элементов нового типа без несущей пространственной частоты, формирующих единственный сфокусированный порядок дифракции, который может занимать всю область восстанавливаемого изображения.

Разработанным методом осуществлен синтез амплитудных бинарных ДОЭ, обладающих малой ошибкой синтеза на уровне 0.1 , а также высокой дифракционной эффективностью на уровне $6.5 \%$.

Результаты оптического восстановления изображений с ДОЭ с использованием микрозеркального модулятора света демонстрируют хорошее качество и высокую по сравнению с голограммами дифракционную эффективность.

\section{Финансирование работы}

Работа выполнена при финансовой поддержке Российского научного фонда (РНФ), грант № 19-19-00498.

\section{Конфликт интересов}

Авторы заявляют, что у них нет конфликта интересов

\section{Список литературы}

[1] Soifer V.A., Doskolovich L.L., Kazanskiy N.L. Methods for Computer Design of Diffractive Optical Elements, Wiley, 2002. $784 \mathrm{p}$.

[2] Путилин А.Н., Морозов А.В., Копенкин С.С., Дубынин С.Е., Бородин Ю.П. // Опт. и спектр. 2020. Т. 128. № 11. C. 1694-1702.

[3] Евтихиев Н.Н., Краснов В.В., Кузьмин И.Д., Молодцов Д.Ю., Родин В.Г., Стариков Р.С., Черёмхин П.А. // Квант. электрон. 2020. Т. 50. № 2. С. 195-196. 
[4] Evtikhiev N.N., Starikov S.N., Shaulskiy D.V., Starikov R.S., Zlokazov E.Y. // Optical Engineering. 2011. V. 50. N 6. P. 065803.

[5] Schnars U., Falldorf C., Watson J., Jüptner W. Digital Holography and Wavefront Sensing. 2015.

[6] Евтихиев Н.Н., Стариков С.Н., Черёмхин П.А., Курбаmова E.A. // Изв. вузов. Радиофизика. 2014. Т. 57. № 8-9. T. 711-727.

[7] Lesem L.B., Hirsch P.M., Jordan J.A. // IBM J. Research and Development. 1969. T. 13. N 2. P. 150-155.

[8] Shimobaba T., Kakue T., Endo Y., Hirayama R., Hiyama D., Hasegawa S., Nagahama Y., Sano M., Oikawa M., Sugie T., Ito T. // Optics Express. 2015. V. 23. N 13. P. 17269.

[9] Cheremkhin P.A., Evtikhiev N.N., Krasnov V.V., Porshneva L.A., Rodin V.G., Starikov S.N. // Proc. SPIE. 2014. V. 9131 . P. 913124.

[10] Chao T.-H., Lu T., Walker B., Reyes G. // Proc. SPIE. 2014. V. 9094. N 5. P. 909402.

[11] Liang J., Kohn R.N., Becker M.F., Heinzen D.J. // Appl. Opt. 2010. V. 49. N 8. P. 1323-1330.

[12] Ren Y.X., Lu R. De, Gong L. // Annalen der Physik. 2015. V. 527. P. 447-470.

[13] Evtikhiev N.N., Zlokazov E.Y., Krasnov V.V., Rodin V.G., Starikov R.S., Cheremkhin P.A. // Quantum Electronics. 2020. V. 50. N 7. P. 667-674.

[14] Gerchberg R.W., Saxton W.O. // Optik. 1969. V. 2. N 2. P. 237-246.

[15] Bondareva A.P., Cheremkhin P.A., Evtikhiev N.N., Krasnov V.V., Rodin V.G., Starikov S.N. // Proc. SPIE. 2014. V. 9216. P. 92161J.

[16] Krasnov V.V. // Proc. SPIE. 2016. V. 10022. P. 1002226.

[17] Fienup J.R. // Appl. Opt. 1997. V. 36. N 32. P. 8352-8357. 